Aus dem Medizinhistorischem Institut der Universität Zürich

(Direktor: Prof. Dr. med. E.H. Ackerknecht)

\title{
Ambroise-Auguste Liébeault (1823-1904) der Begründer der «Ecole hypnologique de Nancy»
}

\author{
Von HaNs H. Walser, Zürich
}

In den letzten Jahrzehnten des vorigen Jahrhunderts erreichte das Interesse für hypnotische Erscheinungen einen seiner Höhepunkte. Wurde das Publikum von der unheimlichen und gefahrvollen Seite der Hypnose besonders angezogen, so eröffneten sich den Ärzten lange Zeit vernachlässigte und darum oft als neu empfundene Möglichkeiten zur Behandlung nervöser Krankheiten und zum Studium körperlich-seelischer Zusammenhänge. Die Untersuchungen von Jean-Martin Charcot (1825-1893) trugen wohl am meisten dazu bei, der Hypnose einen Platz in der zeitgenössischen Medizin zu sichern. Wenige Jahre nachdem Charcot 1878 diese Studien an der Salpêtrière in Paris aufgenommen hatte, entstand aber an der kleinen medizinischen Fakultät von Nancy in Lothringen eine Arbeitsgruppe, die eine modernere Theorie der Hypnose vertrat und die die von Charcot vernachlässigte hypnotische Therapie in den Mittelpunkt stellte. Diese «Schule von Nancy» stand von 1883 an etwa ein Jahrzehnt in Blüte, und wenn es zunächst scheinen mag, sie hätte wenig Dauerndes erreicht, so zeigt eine genauere Betrachtung im Gegenteil, daß ihre mehr indirekten Auswirkungen auf Psychotherapie und Psychiatrie beträchtlich sind und bis in unsere Zeit reichen. Es ist eines der zahlreichen Paradoxa dieser Schule, daß diese Wirkungen hauptsächlich vom deutschen Sprachgebiet aufgenommen wurden.

Die «Schule von Nancy» wurde von der Medizingeschichte bisher eher vernachlässigt. Sie hat erst in den letzten Jahren eine eingehendere zusammenfassende Darstellung erfahren, doch ist diese Arbeit von André CuVELIER leider nicht leicht zugänglich. Im übrigen sind insbesondere die biographischen Angaben über den Begründer wie über die hauptsächlichen Vertreter, H.-M. Bernheim, H. Beaunis und J.LiÉgeois, in kleinen Artikeln in der allgemeinmedizinischen Literatur zerstreut. Ein Zeichen für das wiedererwachende Interesse an der Hypnose und ihrer Geschichte ist es 
wohl, wenn kürzlich in den USA eine biographische Studie über H.-M. Bernheim erschienen ist. Wir beabsichtigen nicht, im folgenden diese historischen Lücken zu schließen, sondern möchten lediglich die Aufmerksamkeit auf die in verschiedener Hinsicht bemerkenswerte Arbeitsgemeinschaft von Nancy lenken. Dazu müssen wir, nach einer Skizzierung der Vorgeschichte des französischen Hypnotismus, auf die Lebensgeschichte von A.-A.LIÉbeault eingehen und werden dann versuchen, seine Lehre und diejenige der «Schule von Nancy» kurz darzustellen, um schließlich auf die fortwirkende Bedeutung derselben hinzuweisen.

\section{Zur Vorgeschichte des Hypnotismus}

Hypnotische Erscheinungen sind uralter Menschheitsbesitz, und sie haben kulturgeschichtlich eine große Rolle gespielt. Erst in der zweiten Hälfte des 18. Jahrhunderts wurde aber von Franz Anton Mesmer (17341815) mit dem «tierischen Magnetismus» ein großangelegter, wenn auch eigenartiger Versuch gemacht, die Hypnose der ärztlichen Therapie dienstbar zu machen. Der Höhepunkt in Mesmers wechselvollem Leben war zweifellos sein Pariser Aufenthalt, der von 1778 bis zur Französischen Revolution dauerte. Seine «Magnetisierungen» erregten auch in den höchsten Gesellschaftskreisen ein sehr lebhaftes Interesse, so daß sich schon 1784 eine königliche Untersuchungskommission darüber auszusprechen hatte. Theorie und Methodik des «Mesmerismus» standen in ausgeprägtem Gegensatz zum medizinischen Zeitgeist der Aufklärung, und der Bericht dieser Kommission fiel auch entsprechend aus. Dies tat aber dem Magnetismus um so weniger Eintrag, als er ohnehin vorwiegend von medizinischen Laien getragen wurde. Einer dieser Schüler Mesmers, der Marquis Chastenet DE Puysegur, machte die wichtige Entdeckung des künstlichen Somnambulismus, und er sorgte außerdem für eine weite Verbreitung der Lehre, indem er in verschiedenen französischen Städten - so auch in Straßburg - «harmonische Gesellschaften» gründete. Die Französische Revolution brachte zwar einen schweren Rückschlag, während der Restauration erholte sich der Mesmerismus aber rasch wieder. Er wurde auch von den deutschen Romantikern begierig aufgenommen, was uns hier aber nicht beschäftigen kann. In Frankreich war die Bewegung so stark, daß sie von den medizinischen Gremien nicht mehr ignoriert werden konnte. Nachdem schon in den 1820er Jahren in Pariser Spitälern von Magnetiseuren Demonstrationen abgehalten worden waren, hatte sich 1831 Henri-MARIE Husson 
(1772-1853) als Präsident einer Kommission der Académie de Médecine über diese Frage zu äußern. Zum sichtlichen Mißbehagen seiner akademischen Kollegen sprach er dabei dem tierischen Magnetismus sowohl einen theoretischen wie einen therapeutischen Wert zu. Die Reaktion ließ nicht auf sich warten, und 1837 erklärte Dubors (d'Amiens) vor derselben Akademie die Magnetiseure entweder als Betrüger oder als Genarrte. Daraufhin scheint die Frage für etwa zwei Jahrzehnte aus der offiziellen medizinischen Diskussion gefallen zu sein. Dies wird verständlicher, wenn man sich vor Augen hält, daß der alte «Mesmerismus» von einem Wust von irrationalen, vorwiegend parapsychologischen Meinungen durchsetzt war (z.B. Hellsehen, Telepathie, Transposition der Sinne u.a.), die oft völlig in den Vordergrund traten. Ein ernsthafter Arzt konnte sich deshalb kaum öffentlich zur "mesmeristischen Therapie» bekennen - es sei denn, er hätte sich als ärztlicher Magnetiseur etabliert und sich seine Patienten außerhalb der offiziellen Medizin gesucht. Ärzte, die auf wissenschaftliche Reputation Wert legten, beschäftigten sich höchstens im geheimen mit diesem anrüchigen Gegenstand: Dies sagt noch 1858 Etienne-Eugk̀ne Azam (1822-1899), immerhin Professor für Medizin in Bordeaux; und Poincaré, der 1866 vor der «Académie de Stanislas » in Nancy einen sonst recht vernünftigen Vortrag über den Hypnotismus hielt, verwahrte sich gegen den Verdacht, praktische Versuche ausgeführt zu haben. Ein Arzt, der sich in jenen Jahren ernsthaft mit dem Hypnotismus beschäftigen wollte, brauchte deshalb nicht nur viel Mut, sondern er mußte auch bereit sein, auf äußeren Erfolg der Sache zuliebe zu verzichten: Und A.-A. LiÉBEAult besaß diese Eigenschaften.

Die frühen Theorien des Mesmerismus trugen nicht dazu bei, diesen für die Medizin annehmbar zu machen. Mesmer selbst war vom physikalischen Magnetismus ausgegangen, hatte dann aber eine eigenartige «kosmobiologische» Lehre aufgestellt, indem er naturwissenschaftliche Gedanken und kosmische Spekulationen auf etwas krause Weise zu einem medizinischnaturphilosophischen System verband. So nahm er an, daß im Weltall ein «Fluidum» existiere, wodurch jeder Mensch mit den Gestirnen wie auch mit seinen Mitmenschen verbunden sein sollte. Dieses Fluidum wurde materiell vorgestellt, es sollte physikalischen Gesetzen gehorchen, polare Richtung haben, akkumuliert und übertragen werden können, es sollte aber außerdem physiologische Wirkungen haben und im menschlichen Körper die gestörte Harmonie wiederherstellen können. In den folgenden Jahrzehnten hielten wohl die meisten Magnetiseure an dieser oder an einer 
ähnlichen Theorie fest. Dabei hatte schon um das Jahr 1815 der portugiesische Abbé Faria bei seinen öffentlichen Demonstrationen in Paris die Ansicht vertreten, ein rein psychischer Einfluß, nämlich die Überzeugung des Magnetisierten, sei die Ursache aller beobachteten Erscheinungen. Von nun an stand eine psychische Theorie, die sich später als siegreich erweisen sollte, neben der älteren Fluidumtheorie. Alexandre Bertrand (17951831) übernahm diese neuen Anschauungen von Faria, und sein Werk von 1823 wird als Anfang des französischen wissenschaftlichen Magnetismus bezeichnet, leider ging es aber in der Flut der mesmeristischen Literatur unter. Auch die zahlreichen Demonstrationen in Laienkreisen zeigten nach wie vor viel Ungereimtes und oft einen Mangel an der einfachsten Kritik man lese etwa die Memoiren des Arztes und Magnetiseurs Alphons Teste, der sich, wie verschiedene ernsthafte Männer, immerhin bemühte, die Spreu vom Weizen zu scheiden. Oder man erinnere sich an den gutmütigen Spott, mit welchem Gustave Flaubert den Mesmerismus bedacht hat. Ein wichtiger Fortschritt kam dann aus dem Auslande, nämlich von JaMES BraId (1795-1860), einem Chirurgen in Manchester, der in seinem Buche von 1843 einen Trennungsstrich zwischen dem Mesmerismus und dem Hypnotismus zog: Letzteren bezeichnete er als eine Methode, das Nervensystem in einen neuen Zustand zu versetzen, welcher für die Heilung gewisser Störungen sehr vorteilhaft sei. Auch er vertrat eine vorwiegend psychische Theorie. Braids Werk blieb in Frankreich unbekannt, bis es E.-E.AzAM 1859 ans Licht zog und in der Folge die Chirurgen Paul Broca und Louis Velpeau dazu brachte, den Hypnotismus vor der Académie de Médecine zu vertreten. A.-A. LiÉBEAult las davon, und diese Mitteilungen gaben den Anstoß, daß er von 1860 an die halbvergessenen Hypnosestudien seiner Studentenzeit wiederaufnahm.

Aber weder seine Bemühungen noch diejenigen von zahlreichen andern Hypnotiseuren genügten, um dem Hypnotismus einen Platz in der Medizin zu sichern. Vielleicht lag es an einem Wechsel des medizinischen Zeitgeistes, vielleicht auch mehr am Eingreifen des großen Charcot, wenn in den 1880 er und 1890er Jahren der Hypnotismus wieder einen Höhepunkt erreichte.

\section{A.-A.Liébeaults Leben und die «Schule von Nancy»}

Ambroise-Auguste Liébeault wurde am 16. September 1823 in einem bescheidenen Bauernhause im Dorfe Favières in Lothringen geboren. Der aufgeweckte Knabe wurde von seinen Eltern zum Priester bestimmt; 
im Alter von zwölf Jahren brachten sie ihn deshalb in ein Seminar. Seine Neigungen galten aber bald mehr den Naturwissenschaften und der Medizin, doch durfte er erst nach dem Tode seines Vaters das Berufsziel ändern. Mit einundzwanzig Jahren schrieb er sich als Medizinstudent in Straßburg ein. Trotz der nur ärmlich ausgestatteten Institute und trotz der Behinderungen durch den wissenschaftlichen Zentralismus Frankreichs, der die beiden außerhalb Paris gelegenen Fakultäten nicht großwerden ließ, war die medizinische Ausbildung damals in Straßburg eine recht gute. Liébeault studierte bei bedeutenden Männern, so beim Kliniker und Pathologen Charles Schutzenberger (1809-1881), bei den Chirurgen Charles Sedillot (1804-1883) und Philippe Rigaud (1805-1885) und bei dem Physiologen und Pathologen Emile Kuss (1815-1871).

Schon während des Studiums wurde Liébeault auf den «tierischen Magnetismus » aufmerksam, und zwar durch einen Krankheitsfall im Spital. Ein Studienkollege lieh ihm ein Buch über diesen Gegenstand, dann las er ein Werk des Pariser Magnetiseurs A.Teste und den Akademiebericht von H.-M.Husson. Seine Lehrer rieten ihm von einer Vertiefung in diese verpönten Probleme ab, aber Liébeault begann nun auf eigene Faust zu hypnotisieren. Da er damit einige Erfolge hatte und schließlich auch eine natürliche Somnambule entdeckte, stand für ihn fest, daß etwas an diesem eigenartigen «Magnetismus» sein müsse.

Zunächst aber promovierte er 1850 mit einer chirurgischen Dissertation, heiratete und ließ sich in Pont-Saint-Vincent bei Nancy als Landarzt nieder. Er erwarb sich eine gute Praxis und dachte zunächst nicht mehr an den Hypnotismus. Einmal aber hatte er ein Mädchen zu behandeln, das an hartnäckigen Krämpfen litt und bei welchem er gerne eine «magnetische Kur» versucht hätte, die sich aber der Vater des Mädchens verbat. Da seine ländlichen Patienten solchen «Neuerungen» überhaupt nicht geneigt waren, zügelte Liébeault seine wissenschaftliche Neugier nochmals. Als er aber 1860 von den Hypnoseversuchen der Chirurgen PAUL Broca und Louis Velpeau las, machte er sich ernsthaft an das Studium des Hypnotismus. Wohl verlor er dadurch gelegentlich einen Patienten, und um einen größeren Anreiz für seine Kuren zu schaffen, übte er diese unentgeltlich aus, während die Medikamente bezahlt werden mußten.

Ein wissenschaftlich ausgebildeter Arzt, der für den Hypnotismus eintreten wollte, mußte damals bereit sein, das Schicksal eines Außenseiters auf sich zu nehmen. Liébeault hatte außerdem eine zwar sanfte, aber unerschütterliche Beharrlichkeit, wozu ein tiefes, etwas einseitiges Interesse 
an rational schwer erklärbaren Lebenserscheinungen kam. Schließlich war er auf fast weltfremde Art gleichgültig gegenüber äußeren Erfolgen. Den Zeitgenossen ist ein prophetischer Zug an ihm aufgefallen, doch war er frei von Proselytenmacherei, so begeistert er den späteren Besuchern auch seine Methode demonstrierte.

Man muß diese Charaktereigenschaften Liébeaults kennen, um verstehen zu können, daß er 1864 seine Landpraxis aufgab, um als «ärztlicher Magnetiseur» nach Nancy zu übersiedeln. Dort hatte er zunächst noch genug Muße, um seine Erfahrungen und Theorien in einem umfangreichen Buche niederzulegen, welches 1866 erschien. Es hatte keinen Erfolg, und während zwanzig Jahren wurden ganze fünf Exemplare davon verkauft. Die Besprechung von A. FoviLLE in den Annales médico-psychologiques mag am meisten zu diesem Mißerfolg beigetragen haben: Foville attestierte dem Verfasser zwar seinen guten Willen, hielt aber seine Gleichsetzung von natürlichem und hypnotischem Schlaf für unrichtig, kritisierte Liébeaults «Nervenkraft», welcher, wie dem «Archäus» van Helmonts, gar zu viele Aufgaben überbürdet worden seien, und bemängelte schließlich die allzu einfachen Vorstellungen Liébeaults vom Heilungsmechanismus. Foville verlangte für die Pathologie eine sichere physiologische Grundlage, während Liébeault, der sich für theoretische Medizin nicht interessierte, lediglich die um die Jahrhundertmitte üblichen physiologischen Anschauungen benützt hatte. Foville ging aber gewiß zu weit, wenn er deshalb dem therapeutischen Vorgehen Liébeaults das Vertrauen absprach.

Wohl hatte Liébeault nach seiner Übersiedlung versucht, seine Behandlungsmethode bei den Ärzten bekanntzumachen, er wurde aber zurückgewiesen, wohl auch der Scharlatanerie verdächtigt und gelegentlich verlacht, so daß er sich zurückzog und wenig Kontakt mit seinen ärztlichen Kollegen unterhielt. BernheIM sagte später einmal, er habe nach fast zehnjährigem Aufenthalt in der Kleinstadt Nancy den Namen Liébeaults noch nie gehört gehabt. Auch seitens der Patienten hatte Liébeault wenig Unterstützung zu erhoffen, denn er behandelte meist bescheidene Leute vom Land, Kleinbürger aus Nancy und arme Arbeiter.

Während des Deutsch-Französischen Krieges kehrte Liébeault vorübergehend zu seiner alten ärztlichen Tätigkeit zurück, indem er als Arzt in einem Militärspital wirkte. Die indirekten Folgen dieses Krieges sollten aber schließlich für sein Leben entscheidend werden: Mit der Niederlage hatte Frankreich eine seiner drei medizinischen Fakultäten verloren, und 1872 beschloß die Nationalversammlung die Verlegung der Straßburger 
Fakultät nach Nancy. Die meisten der Professoren folgten diesem Rufe, so daß Nancy nach einigen Jahren des Aufbaues ein nicht unbedeutendes medizinisches Zentrum wurde. Trotzdem lebte Liébeault noch weitere zehn Jahre in Abgeschiedenheit dahin, bis er von einzelnen Mitgliedern der Fakultät recht eigentlich «entdeckt» wurde: Ein Doktor Dumont, der etwas außenseiterische Neigungen hatte, damals aber als «Chef des travaux physiques» der Fakultät angehörte, ließ sich von Liébeault in die Praxis des Hypnotismus einführen, und dank seiner Beziehungen konnte er in der Irrenanstalt Maréville bei Nancy weitere Versuche machen und diese am 10. Mai 1882 vor der angesehenen «Société de Médecine» demonstrieren. Die meisten der etwa dreißig anwesenden Ärzte - darunter auch Bernheim blieben zwar skeptisch, viele aber waren doch einigermaßen beeindruckt.

Hippolyte-Marie Bernheim (1837-1919) war damals bereits ein anerkannter Meister der internen Klinik. Er war aus der Straßburger Fakultät hervorgegangen und hatte dann in Paris und insbesondere bei Rudolf VIrchow in Berlin studiert. Dort hatte er gesehen, wie sehr die naturwissenschaftlich orientierte deutsche Medizin der französischen überlegen war, und er bemühte sich, die neuen exakten Anschauungen in Frankreich einzuführen. Ein solcher Mann mußte dem Hypnotismus von Anfang an skeptisch gegenüberstehen. Bernheim hatte sich nun in jenen Jahren dem Studium der organischen Nervenkrankheiten zugewandt und hatte dabei die Wirkung des Eisenmagneten auf Lähmungen untersucht. Damit geriet er in die Náchbarschaft zum «tierischen Magnetismus», der ja auch aus der Beschäftigung mit physiologischen Wirkungen des Eisenmagneten hervorgegangen war - freilich unter ganz anderen zeitlichen und wissenschaftlichen Voraussetzungen. Daß der moderne Kliniker Bernheim trotzdem den Schritt zum Hypnotismus tat und ihm seine Klinik öffnete, zeigt, wie unabhängig und wie weitblickend er als Wissenschafter war. Für die Sache des Hypnotismus war Bernheims Haltung ein wahrer Glücksfall, denn erst er als anerkannter Kliniker konnte Liébeaults Werk wirkliche Anerkennung verschaffen.

Gleichzeitig mit Bernheim interessierten sich zwei weitere Professoren für Liébeaults Arbeit: der Jurist Jules LiÉgeors und der Physiologe Henri Beaunis (1830-1921). Beaunis war als Militärarzt ausgebildet, hatte an der Straßburger Fakultät bis zum Deutsch-Französischen Kriege Physiologie gelehrt und war dann ebenfalls nach Nancy übersiedelt, wo er den physiologischen Unterricht einführte. Als Schüler von Emile Kuss war er mit der damals modernsten deutschen Physiologie sehr gut vertraut, 
außerdem hatte er psychologische Interessen. Er studierte besonders die psycho-physischen Phänomene des Hypnotismus, z.B. die Einflüsse auf Herzaktion, Muskelkraft usw., und seine Untersuchungen waren im Kampfe um den Hypnotismus besonders wichtig, da der Beweiswert körperlicher Veränderungen bei vielen Zweiflern größer war als derjenige «bloßer» psychischer Erscheinungen. Aufsehen erregte insbesondere das Erzeugen von Hautblasen durch Suggestion während des hypnotischen Zustandes, was dem Apotheker Focachon aus Vézelise in Zusammenarbeit mit Beaunis und Liébeault gelang: Damit wurde eine rationale Erklärung der Stigmatisation möglich, welch letztere gerade damals durch den Fall der Louise Lateau die Gemüter erregte.

Der Jurist Jules LiÉGeois (1823-1908) arbeitete sich wohl zunächst aus psychologischem Interesse in die Probleme des Hypnotismus ein, widmete sich aber dann besonders der kriminalistischen Seite dieser Erscheinungen. Nach zahlreichen Versuchen schloß er sich der Ansicht Liébeaults an, der Hypnotisierte funktioniere wie ein Automat in der Hand des Hypnotiseurs, er sei also grundsätzlich auch zu verbrecherischen Zwecken zu gebrauchen, wofür die Verantwortung dann allein dem Hypnotiseur zufallen würde. Im April 1884 trug er seine Ergebnisse der «Académie des sciences morales et politiques» in Paris vor, wobei er auf heftigen Widerspruch stieß, der nicht nur dem forensischen Aspekt der Frage, sondern der «Schule von Nancy» und teilweise dem Hypnotismus überhaupt galt. Bei der Verteidigung kam es Liégeois sehr zustatten, daß man in Nancy sehr zahlreiche Hypnoseprotokolle besaß, wie sie außer Liébeault damals wohl niemand vorweisen konnte.

Mit Recht hat man darauf hingewiesen, daß es eine «Schule von Nancy» als Schülergruppe eines Meisters oder als Institution nicht gab. Der bescheidene, unauffällige Liébeault hätte sich nicht zur Lehrergestalt geeignet, seine «Schüler» waren bedeutender als er und besaßen bereits ihren wissenschaftlichen Ruf, und schließlich ließen seine Meinungen und seine Selbstkritik oft zu wünschen übrig. Die große Anhänglichkeit und Verehrung, die ihm seine «Schüler» bewahrten, ist aber ein Beweis mehr, daß ohne Liébeault in Nancy nie eine Hypnoseschule entstanden wäre. Umgekehrt wäre er ohne seine "Schüler» heute ebenso vergessen wie zahlreiche andere ärztliche Hypnotiseure jener Zeit. Ein weiterer glücklicher Zufall war, daß sich in Nancy Gelehrte der verschiedenen Richtungen zusammenfanden: Der Jurist, der Physiologe und der Kliniker waren wirklich berufen, je eine der wichtigsten Seiten des hypnotischen Problems mit Autorität zu ver- 
treten. Hoch ist übrigens der menschliche Anstand dieser Professorengruppe zu preisen: Keiner dieser Männer versuchte je, das Verdienst des kleinen Praktikers zu schmälern, sondern sie freuten sich an seinem Erfolg. Bernheim gelang es auch, Liébeault gelegentlich in taktvoller Weise auf unwissenschaftliche Abweichungen aufmerksam zu machen, ebenso korrigierte er die «Beweise» des sonst verdienstvollen Apothekers Focachon von der angeblichen Fernwirkung der Medikamente, indem er den suggestiven Mechanismus dieses Effektes aufklärte - kurz, er war nicht nur der wichtigste Vertreter, sondern auch der «gute Geist» der Schule von Nancy.

Mit dem Beginn der 1890er Jahre verlor sich der Impetus der ersten Zeit allmählich - sicher nicht zuletzt, weil der Hypnotismus nun immer mehr Anerkennung fand. Liébeault gab 1891 altershalber seine Praxis auf und verfaßte nur noch gelegentlich einen Artikel für die Revue de l'hypnotisme. Beaunis verließ Nancy nach seiner Emeritierung im Jahre 1894, und Bernheim wandte sich zu Liébeaults Betrübnis immer mehr der Wachsuggestion zu, wobei er durch Paul Dubors in Bern allmählich in den Hintergrund gedrängt wurde. Die autochthone, im Sinne der Lehre aber nicht legitime Fortsetzung durch EmILe CouÉ hätte Liébeaults Billigung gewiß nicht gefunden, denn Coué ging noch über die Wachsuggestion Bernheims hinaus und wollte sogar den hypnotisierenden oder suggerierenden Arzt überflüssig machen.

Am Ende seines öffentlichen Wirkens, im Jahre 1891, bereiteten Freunde dem betagten Liébeault eine Feier. Liébeault bekannte dabei, daß er ein glückliches Leben gehabt habe. Die Hauptursache dieser Zufriedenheit lag sicher in seinem freundlichen, bescheidenen Charakter, doch machte ihm auch die späte Anerkennung seines Lebenswerkes große Freude. «Mr.Liébeault va bien et est l'homme le plus heureux de la terre depuis que nous avons rendu justice à son œuvre», schrieb Bernheim am 25. Mai 1887 an Forel. Bei jener Feier erhielt Liébeault eine Bronzeplastik von Mercié zum Geschenk, David als Besieger Goliaths darstellend: Ein Sinnbild für das erfolgreiche Auftreten des lothringischen Praktikers gegen die Schule der Salpêtrière. Auf der Gratulantenliste standen Namen wie Bérillon, Dejerine, Dumontpallier, Milne Bramwell, Krafft-Ebing und Forel; Freud, der bereits eigene Wege ging, fehlte. Die folgenden Lebensjahre Liébeaults scheinen recht ruhig gewesen zu sein. Im Herbst 1902 wurde an seinem Geburtshaus eine Gedenktafel enthüllt, er selbst konnte aber wegen Kränklichkeit und schlechten Wetters den Weg in das entlegene Dorf nicht mehr machen. Umsorgt von Gattin und Adoptivtochter, starb er 1904. Zwei Jahre später 
wurde in der «Ecole de psychologie» in Paris seine Büste aufgestellt, 1935 erhielt er ein Denkmal im Parc Olry in Nancy.

\section{Das Werk A.-A.Liébeaults}

Am Anfang der literarischen Tätigkeit Liébeaults steht sein 1866 erschienenes Hauptwerk: Du sommeil et des états analogues considérés surtout au point de vue de l'action du moral sur le physique. Es wurde erst zwei Jahrzehnte später überhaupt beachtet, erlebte dann 1889 einen teilweisen Neudruck und 1892 sogar eine deutsche Übersetzung, wurde aber nicht einmal von dem wohlmeinenden Bernheim besonders geschätzt. Liébeault hat sodann ein weiteres, kleineres Buch, eine Broschüre und etwa zwanzig Zeitschriftenartikel verfaßt, die alle in Organen des Magnetismus oder Hypnotismus erschienen. Seine Ansichten hat Liébeault in den etwa vier Jahrzehnten seiner Beschäftigung mit dem Hypnotismus kaum geändert, so daß seine Schriften gesamthaft anhand des Hauptwerkes besprochen werden können.

Geschichtlich gesehen steht Liébeault zunächst in der Tradition des französischen ärztlichen Magnetismus, an dessen Schriften er sich autodidaktisch geschult hatte. Er erwähnt deshalb oft Autoren wie Bertrand, Lafontaine, Teste, Charpignon, Chardel, deren wissenschaftliche Haltung ja bezüglich Selbstkritik verschieden war. So wird man sich nicht wundern, wenn die Widerlegung bzw. die rationale Erklärung der verschiedenen parapsychologischen Erscheinungen bei ihm einen recht großen Raum einnimmt. Er erklärte nur das Gedankenlesen als vielleicht möglich, zog aber den rationalen Deutungsversuch von MAURY immerhin vor. Man hat überhaupt manchmal den Eindruck, Liébeault habe gegen einen gewissen Hang zum Mystizismus anzukämpfen gehabt, und ist deshalb wenig verwundert, wenn er sich in den 1880 er Jahren nicht nur über Prophetie und Gedankenübertragung in eher zustimmendem Sinne äußerte, sondern auch mit magnetisiertem Wasser und durch Handauflegen therapeutische Erfolge erzielt haben wollte. Hier kam die alte mesmeristische Erbschaft nochmals zum Durchbruch, und Liébeault wurde selbst zum Opfer einer Autosuggestion, die er bei andern so gut zu erklären wußte. Er hielt auch immer daran fest, daß durch psychische Einwirkungen auf die werdende Mutter körperliche Veränderungen beim Kinde erzielt werden könnten, und er verwandte auf dieses Problem viel Zeit und Mühe. Im ganzen darf man aber doch sagen, daß dieses parapsychologische Beiwerk Liébeaults Behandlungsmethode jedenfalls kaum geschadet hat. 
Sodann knüpfte Liébeault an die psychophysischen Forschungen eines Cabanis und eines Bichat an, die in der ersten Hälfte des Jahrhunderts die französische Medizin tiefgehend beeinflußt hatten. Diese Ansichten dienten ihm als Grundlage zu seiner Theorie, und er wollte eine neue psychophysische Medizin geben, indem er den Einfluß des « Gedankens» auf den Organismus untersuchte, und zwar durch das Studium der passiven Modi des Lebens, also des Schlafes und der analogen Zustände. Diese selbst sollten durch eine psychische Wirkung zustande kommen.

So postulierte er zunächst eine Nervenkraft oder Schöpfungskraft, die im animalen Leben bewußt, im nutritiven Leben unbewußt wirkt. Sie sollte vom Gehirn ausgehen, sich im Nervensystem verteilen, wobei eine Anhäufung die Funktion des betreffenden Organes verbessern, eine Verdünnung sie abschwächen würde. Diese Verschiebung kann durch psychische Einflüsse (insbesondere durch bewußte Gedanken) erfolgen. Konzentriert sich eine Person auf die Idee des Einschlafens, so fließt die Nervenkraft ins Gehirn zurück und wird passiv, sie steht dann in den Sinnes- und Bewegungsorganen nur noch in geringem Maße zur Verfügung. Während des Schlafes werden Sinneseindrücke deshalb höchstens noch ganz unklar wahrgenommen, und die Bewegungen des Körpers sind fast aufgehoben.

Empfindung, Wahrnehmung und Gedächtnis kommen gleichfalls durch die Nervenkraft zustande. Durch diese können latente Erinnerungsbilder wieder erweckt werden, woraus sich das Wiederauftauchen vergessener Eindrücke in Hypnose erklärt, denn während dieser ist ja (wie während des Schlafes) die Nervenkraft im Gehirn angehäuft. Hypnose und Schlaf sind wesensgleich, d.h. sie unterscheiden sich nur dadurch, daß bei jener immer eine Verbindung zum Hypnotiseur besteht (der «Rapport»), während eine solche im Schlafe fehlt oder doch inkonstant ist. Den zentripetalen Wahrnehmungen stehen die zentrifugalen Halluzinationen gegenüber; beide sind für das Individuum gleichwertig (Freud führte später zur Unterscheidung, um welche sich Liébeault nicht weiter bemühte, den Begriff der «Realitätsprüfung» ein). Denken bedeutet das Wirkenlassen der Nervenkraft auf die von den Sinnesorganen gelieferten Materialien.

Der Organismus ist der Vermittler zwischen dem denkenden Wesen und der Außenwelt, und, da der «Gedanke» den Körper auf bewußtem oder unbewußtem Wege verändern kann, ist er der konkrete Ausdruck des «Gedankens». Die Nervenkraft ist im vegetativen wie im animalen System dieselbe, und da im Gehirn Sinneserregungen und unbewußte Körperempfindungen zusammenfließen, so läßt sich annehmen, daß durch den be- 
wußten Gedanken Einflüsse auf das vegetative Leben, d.h. auf alle Körperfunktionen, möglich sind. Dazu muß sich der Mensch aber im hypnotischen Zustande befinden - daran hielt Liébeault immer fest, und er lehnte deshalb wachsuggestive Methoden als ungenügend $a b$.

Sehr zahlreiche, irgendwie mit der Hypnose in Zusammenhang stehende Fragen wurden von Liébeault ebenfalls untersucht. So finden sich Bemerkungen über eine Psychologie der Masse, über Tradition und Meinungsbildung sowie Ansätze zu einer Religionspsychologie, schließlich auch tierpsychologische Beobachtungen in seinem Werk. Wichtiger als alle Theorien wurde aber die reiche Kasuistik. Hier fühlte sich Liébeault als praktischer Arzt zu Hause, und seine sehr zahlreichen Behandlungsprotokolle, von denen er nur einen kleinen Teil publizierte, gaben der "Schule von Nancy» einen starken Rückhalt. Sie betrafen größtenteils innere Krankheiten der verschiedensten Art, darunter viele, die für eine hypnotische Behandlung sicher nicht geeignet waren. $\mathrm{Daß}$ die Hysterie, die Liébeault einmal «maladie du sommeil par excellence» nannte, für seine Methode besonders geeignet war, sah er wohl, zog aber keine weitergehenden Schlüsse daraus. Für die praktische Psychiatrie interessierte er sich nur im Rahmen der Allgemeinpraxis, also nicht besonders stark, so daß er zur Psychopathologie keine der Rede werten Beiträge geliefert hat. Kleine chirurgische Eingriffe und Geburten versuchte Liébeault ebenfalls durch Hypnose schmerzlos zu machen, ohne aber über einige mäßig erfolgreiche Versuche hinauszugelangen.

Überhaupt wirkte Liébeault viel mehr durch sein praktisches Beispiel als durch irgendeine seiner Schriften. Seine Methode entwickelte er gemäß seinen Erfahrungen aus den Angaben der früheren Magnetiseure. Er gelangte schließlich zu einer suggestiven Einleitung der Hypnose, d.h. er suggerierte die körperlichen Symptome des beginnenden Schlafes (Müdigkeit, Schwere der Augenlider, Erlöschen der Sinnesempfindungen usw.). War die Hypnose genügend tief - er beschrieb die verschiedenen Tiefegrade öfters -, dann wiederholte er mit monotoner, aber eindringlicher Stimme allgemeine Suggestionen des Wohlbefindens und der ungestörten Körperfunktionen. Man sieht leicht, daß er kein vertieftes psychologisches Verständnis des einzelnen Patienten anstrebte, sondern vielmehr eine Beseitigung der Krankheiten oder auch bloß der Symptome bei einer größeren Zahl von Kranken in kurzer Zeit. Das unterscheidet seine Methode hauptsächlich von der modernen, tiefenpsychologisch orientierten Psychotherapie. Immerhin mag auch sein Vorgehen selbst heute noch in geeigneten Fällen Gutes stiften. Nicht vertretbar ist dagegen sein Verzicht auf eine vor- 
herige genaue Untersuchung, selbst auf eine Diagnose, und dann sein wenig inidvidualisierendes Suggerieren. Doch schrieb ein recht kritischer Augenzeuge, Liébeaults Hypnotisieren habe trotzdem nicht schematisch gewirkt, und dies muß wohl auf seine Persönlichkeit zurückgeführt werden, die bei aller Bescheidenheit wohl eine beträchtliche «Strahlungskraft» auf die Patienten ausübte.

\section{Der Hypnotismus von Nancy und die Schule der Salpêtrière}

Der Streit zwischen der Studiengruppe von Nancy und der Schule Charcots über Wesen und Diagnose der Hypnose hat seit je ein besonderes Interesse gefunden. Worum ging es dabei eigentlich?

Die wissenschaftlichen Differenzen werden verständlicher, wenn man sich die Verschiedenheit der Ausgangspunkte vor Augen hält: Charcot war Neuropathologe und hatte eine große Klinik zu seiner Verfügung, deren Weltruf er begründet hatte, Liébeault ging vom zeitgenössischen Magnetismus aus und studierte die Hypnose an den ambulanten Patienten seiner ländlichen oder kleinstädtischen Allgemeinpraxis. Charcot kam über das Studium der Hysterie zur Hypnose - und es hatte schon viel bedeutet, daß sich der exakte Kliniker überhaupt der Hysterie zugewandt hatte, geschweige denn, daß er sich nun auch noch mit der Hypnose abgab! - Liébeault aber war durch die Notlage seiner nervösen bäuerlichen Kranken zur hypnotischen Therapie veranlaßt worden. Charcot war natürlich wissenschaftlich dem Praktiker aus der Provinz weit überlegen, und es entbehrt nicht des Reizes, daß Liébeaults Ansichten - freilich veredelt durch die Bemühungen seiner «Schüler» - sich schließlich doch als richtiger erwiesen.

Charcot hielt auch in der Folge daran fest, daß die Hypnose ein krankhafter Zustand sei, welcher nur bei Hysterie überhaupt auftreten könne. Als meisterhafter Kliniker wandte er seine neuropathologische Methode die ihm bei organischen Krankheiten solchen Ruhm gebracht hatte - auch auf das Studium der Hypnose an. Er bezeichnete sie als Zustand «sui generis » und beschrieb 1882 den "grand hypnotisme» als ein dreistufiges Geschehen, bestehend aus Katalepsie, Lethargie und Somnambulismus - drei abgegrenzte Erscheinungsformen, die durch geeignete Manipulationen nacheinander erzeugt werden können und die fixe, somatische, von der Suggestion unabhängige Zustände darstellen. Die Diagnose des «grand hypnotisme» schloß deshalb die Möglichkeit der Simulation aus. Charcot sah natürlich auch, daß sich nicht alle hypnotischen Erscheinungen mit dem «grand hypnotisme» zur Deckung bringen ließen, er grenzte die atypi- 
schen Formen als "petit hypnotisme» ab, welch letzterer die Möglichkeit des Simuliertwerdens offen ließ. Die Frage der Simulation hatte bei den Anschauungen Charcots ein um so größeres Gewicht, weil er der Suggestion wenig Bedeutung beimaß - und speziell der ungewollt vom Hypnotiseur ausgehenden Suggestion zu wenig Beachtung schenkte. Die Simulation war auch praktisch für forensisch-medizinische Fragen von größter Wichtigkeit: Die damalige Pariser Autorität, Paul Brouardel, schloß sich Charcot an. Er erklärte also, Verbrechen in Hypnose könnten nur von Hysterischen begangen werden, und es sei nicht möglich, einen Hypnotisierten zu einem Verbrechen zu bringen, welches er im wachen Zustande keinesfalls auch begehen könnte.

Die «Schule von Nancy» erklärte dagegen, die Hypnose sei eine normalpsychologische Erscheinung, die bei fast allen Menschen hervorgerufen werden könne. Die Suggestion spiele dabei die wichtigste Rolle und das jeweilige hypnotische Zustandsbild sei von der Art der Suggestionen abhängig; eine Hypnose als Krankheitsbild sui generis existiere deshalb nicht. Charcots Erscheinungsformen des "grand hypnotisme» kämen deshalb durch unbewußte Suggestionen seitens des Hypnotiseurs oder durch Imitation unter den Hypnotisierten zustande. Dementsprechend spielte auch die Simulation keine so wichtige Rolle. Bezüglich der Möglichkeit hypnotisierter krimineller Handlungen hielten wenigstens Liébeault und Liégeois daran fest, daß zahlreiche Menschen durch posthypnotische Suggestionen auch gegen Ihren Willen zu Verbrechen veranlaßt werden könnten.

Im Verlauf weniger Jahre zeigte es sich, daß die Wahrheit zum größeren Teile auf der Seite von Nancy war, ausgenommen bei der Frage der kriminellen Suggestionen. Dazu kam, daß Nancy viel größeres Gewicht auf die eigentliche hypnotische Therapie legte, so daß man versteht, daß sich die ausländischen Ärzte nach ihren Studien an der Salpêtrière nach Nancy wandten (so J.Delboeuf, Albert Moll, Sigmund Freud, Dejerine u.a.).

Persönliche Motive trugen zur Verschärfung des Kampfes bei: Charcot ertrug es nur schwer, daß ein kleiner Landpraktiker mehr Recht haben sollte als er, und mit ihm scheinen zahlreiche seiner Anhänger dies als Kränkung empfunden zu haben. Paul Janet trat wohl aus ähnlichen Gründen gegen Jules Liégeois, den Juristen aus einer kleinen Provinzfakultät, auf. Man darf auch nicht vergessen, daß in den medizinischen Gremien jener Zeit noch immer eine gewisse Kampfeslust aus der jahrzehntelangen Abwehr gegen den alten Magnetismus steckte. Natürlich fehlte es auch nicht am Vorwurf ungenauer Beobachtung und ungenügender 
Kritik: Dazu wäre zu sagen, daß sich wohl alle Untersucher, Charcot und Liébeault nicht ausgenommen, gelegentlich gröblich täuschen ließen.

\section{Die Auswirkungen der «Schule von Nancy»}

Erinnern wir uns zunächst daran, daß die «Schule von Nancy » vor allem durch das praktische Beispiel wirkte: Sie betrachtete die Hypnose nicht als einen beliebigen Wissensgegenstand, sondern als therapeutische Methode, die am Kranken selbst gesehen, erlebt und schließlich selbst geübt werden mußte. So ging der hauptsächliche Einfluß von der inneren Klinik Bernheims - und in geringerem Maße auch von der Praxis Liébeaults aus, wo die ausländischen Ärzte die Hypnose sahen und praktisch erlernten. Wir können hier nicht die ganze Ausstrahlung dieser Unterrichtsstätten nach Großbritannien, Belgien, Schweden, Deutschland, Österreich und nach andern Ländern untersuchen, sondern möchten nur auf zwei - allerdings wichtige - Entwicklungslinien aufmerksam machen. Jede derselben ging von einem der prominentesten Ärzte aus, die sich in Nancy in der Praxis der Hypnose unterrichten ließen; es waren Auguste Forel und Sigmund Freud.

Auguste Forel (1848-1931) war damals Professor für Psychiatrie in Zürich. Da er in Deutschland psychiatrisch ausgebildet war, vertrat er zwar eine vorwiegend hirnanatomisch orientierte Anstaltspsychiatrie, doch hatte er andererseits seit seiner frühen Jugendzeit ein lebhaftes Interesse an psychophysischen Zusammenhängen. Zudem war sein Großvater einer der vielen französischen Laienmagnetiseure gewesen, und als Knabe hatte er von dessen Kuren gehört. Sein Besuch in Nancy im März 1887 bedeutete für ihn eine wahre Erleuchtung: «Ich konnte mich nicht genug über alles Gesehene wundern ... alles wurde mir sonnenklar, und es fiel mir wie Schuppen von den Augen », schrieb er später darüber. Klar wurden ihm eben jene langgesuchten Zusammenhänge zwischen Körper und Seele, die ihn später zum Monismus führen sollten. Noch folgenreicher wurde wohl die Einführung der Hypnose als therapeutische Methode in die praktische Psychiatrie: Forel führte gerade in jenen Jahren die Totalabstinenz zur Behandlung der Alkoholkranken ein, und wenn er bei diesen durch die Hypnose auch keine allzu großen Erfolge erzielen konnte, so bewährte sie sich doch aufs beste bei neurotischen Zuständen. Jedenfalls war durch die hypnotische Behandlung eine weitere Bresche in den therapeutischen Nihilismus der zeitgenössischen Psychiatrie geschlagen. Natürlich versuchte Forel die Hypnose auch bei eigentlichen Geisteskrankheiten, doch mußte 
auch er sehen, daß dies wenig aussichtsreich war. Seit Forels Zeit zeichnete sich aber die «Zürcher Schule» der Psychiatrie durch ein besonderes therapeutisches Interesse aus. In theoretisch-psychiatrischer Hinsicht wandte sich Forel unter dem Eindruck des Hypnotismus entscheidend von einer rein somatischen Theorie ab, wenn er den körperlichen Anteil der psychischen Phänomene auch im Rahmen seines Monismus weiter anerkannte. Trotzdem war damit eine Wendung zu einer mehr psychischen Theorie der Geisteskrankheiten gegeben, was besonders bei Forels Schülern zum Ausdruck kommen sollte: Adolf Meyer (1866-1950) schuf eine psychobiologische Theorie der Geisteskrankheiten, und vertrat sogar bei der Schizophrenie eine psychische Ätiologie. Eugen Bleuler (1857-1939) studierte dieselbe Krankheit mit einer psychologisch-verstehenden Methode, die sich als so überlegen erwies, daß sein Werk von 1911 heute noch als grundlegend gilt. Ohne die hypnotische Schulung bei Forel hätte sich Bleuler wohl auch der Psychoanalyse gegenüber nicht so aufgeschlossen gezeigt, die er ja als einziger bedeutender Psychiater deutscher Zunge sehr früh in ihrem Wert erkannte. Forel selbst lehnte allerdings die Psychoanalyse ab, was um so auffälliger ist, als er die Grundlagen für ihr Verständnis in reichem Maße besessen hätte.

Sigmund Freud (1856-1939) ging im Sommer 1889 für einige Wochen nach Nancy, um seine hypnotische Technik zu vervollkommnen. Auguste Forel hatte ihm eine Empfehlung an Bernheim mitgegeben, und diesem gefiel der junge Wiener Arzt, der bereits eines seiner Bücher ins Deutsche übersetzt hatte, sehr gut. Freud hatte im Winter 1885/86 bei Charcot studiert und hatte die Hypnose dann in seiner Privatpraxis angewandt. Es gelang ihm aber nicht, alle Kranken zu hypnotisieren und immer die gewünschte Tiefe der Hypnose zu erzielen. An Bernheims Patienten beeindruckte ihn am tiefsten die Möglichkeit starker seelischer, dem Bewußtsein aber unbekannter Vorgänge. Bernheims Beobachtung, daß die Erinnerung an Vorgänge während tiefer Hypnose nur scheinbar ganz aufgehoben ist, wurde für Freud später entscheidend wichtig: Als er später die «Katharsis» (das «Abreagieren») als Methode verbessern wollte, überlegte er sich, daß die Patienten doch eigentlich all das «wissen» müßten, was ihnen sonst nur in Hypnose zugänglich ist. Aus dieser Überlegung entstand schließlich das «freie Assoziieren», welches noch heute die Grundlage der psychoanalytischen Technik bildet. Vom eigentlichen Hypnotismus behielt Freud dagegen die Lagerung des Patienten auf einem Ruhebett bei. Auch in theoretischer Hinsicht wurde Freud durch die «Schule von Nancy» be- 
einflußt: Liébeault vertrat eine «energetische Theorie», und der Energiebegriff lag Freud seit seinen Studien in BRüCKES physiologischem Laboratorium bereits sehr nahe. Freilich läßt sich Liébeaults «force nerveuse» ebensowenig ohne weiteres mit Freuds «Libido » gleichsetzen wie Liébeaults «unbewußte Vorgänge» mit dem «Unbewußten» der Psychoanalyse. Freud konnte manche wesentlichen Ansichten, z. B. solche über den Mechanismus des Einschlafens, über das Zustandekommen der Halluzinationen, über die Wichtigkeit des Schlafwunsches, in Liébeaults Buch finden, das er zumindest in der gekürzten Ausgabe von 1889 kannte, und er war sich des Einflusses Liébeaults, wie eine briefliche Äußerung zur «Traumdeutung» zeigt, durchaus bewußt. Schließlich ist auch Liébeaults Theorie, bei aller Berücksichtigung der körperlichen Vorgänge, doch psychologisch (und - nicht oder weniger - «physiologisch»), und Freud gab später den Versuch, seine psychologischen Funde mit Körperstrukturen zu verbinden, ebenfalls bald endgültig auf. Im übrigen muß wohl nicht besonders betont werden, daß Freud seit den 1890 er Jahren weit über die Hypnoselehre hinausging, doch gehört das, was er in Nancy lernte, unzweifelhaft mit zu den wichtigsten Grundlagen der Psychoanalyse.

Zusammenfassend läßt sich sagen, daß die «Ecole hypnologique» von Nancy ihren Ursprung in der Lebensarbeit des lothringischen Landpraktikers A.-A. Liébeault hat, welcher sich die Hypnose autodidaktisch aus den Schriften des umstrittenen Magnetismus aneignete, von 1860 an mit großer Hingabe hypnotische Kuren durchführte und eine psychologische Theorie der Hypnose vertrat, die sich später sogar den Lehren des großen Charcot überlegen zeigte. Liébeaults Hauptinteresse galt der Therapie, das kritische, wissenschaftliche Arbeiten war nicht seine Stärke, und er wäre heute wohl vergessen, wenn nicht H.-M. Bernheim und H.Beaunis, beides Professoren an der Medizinischen Fakultät in Nancy, seine Behandlungsmethode und seine Theorie zeitgemäß ausgebaut und mit dem Juristen J. Liégeois zusammen vor der wissenschaftlichen Welt vertreten hätten. Von 1883 an wurde namentlich die internistische Klinik von H.- M. Bernheim von zahlreichen, meist ausländischen Ärzten besucht, und die psychologische (bzw. suggestive) Hypnosetheorie breitete sich zusammen mit der hypnotischen Behandlung von Nancy weit über Europa aus. Dies stellt den eigentlichen Beginn der wissenschaftlichen, modernen Psychotherapie dar. Von den weitern Entwicklungslinien schienen diejenigen im deutschen Sprachgebiet von besonderer Bedeutung: Durch A.Forel und seine Schüler E. Bleuler 
und Adolf Meyer (in den USA) erhielt der Hypnotismus schließlich Einfluß auf die (klinische) Psychiatrie, andererseits wurden die Lehren der «Schule von Nancy» durch S. Freud zu einer der wichtigsten Grundlagen der Psychoanalyse. Liébeaults Leben ist ein Beispiel dafür, wie die treue Hingabe eines einzelnen, einfachen Arztes an eine besondere ärztliche Aufgabe schließlich tiefgehende und dauernde Wirkungen auf den Gang der Medizingeschichte haben kann.

\section{Literatur (Auswahl)}

${ }^{1}$ E. H. Ackerknecht, Kurze Geschichte der Psychiatrie, Stuttgart 1957.

${ }^{2}$ H. M. Bernheim, Die Suggestion und ihre Heilwirkung, deutsch von Sigmund Freud, Leipzig und Wien 1892.

${ }^{3}$ A. Cuvelier, L'Ecole hypnologique de Nancy, Diss. med., Nancy 1953.

${ }^{4}$ O. Diethelm, Adolf Meyer (in K. Kolle, Große Nervenärzte, Band II, Stuttgart 1959).

5 E. Jones, Das Leben und Werk von Sigmund Freud, Band I, Bern und Stuttgart 1960.

${ }^{6}$ P. Kissel, L'Ecole neuro-psychiatrique de Nancy, Médecine de France 11 (1955) No. LXVIII.

7 A.-A. Liébeault, Du sommeil et des états analogues, Paris 1866.

${ }^{8}$ Revue de l'hypnotisme, Bände I-IX (1886-1895).

${ }^{9}$ Revue médicale de l'est XI (1882) ff.

${ }^{10}$ H. H. Walser, Aus Auguste Forels Briefwechsel (in Vorbereitung).

Die vorliegende Arbeit ist die umgearbeitete Fassung eines Referates, welches ich an der Henry-E.-Sigerist-Tagung in Luzern im Frühjahr 1960 halten durfte (Sekretär: Dr. med. Ch. Salzmann). Bei der Beschaffung des Materials haben mich die Herren Prof. Dr. med. P. Kissel und Dr. med. A. Cuvelier sowie Frau G. Koest, Bibliothekarin (alle in Nancy), sowie Herr Michelet, alt Schuldirektor in Favières, auf die liebenswürdigste Weise unterstützt. Herrn Professor E. H. ACKerknecht bin ich für zahlreiche Anregungen verpflichtet. Ihnen allen sei herzlich gedankt. 